\title{
A RESIDUAL-BASED HP-MESH OPTIMIZATION TECHNIQUE FOR PETROV-GALERKIN SCHEMES WITH OPTIMAL TEST FUNCTIONS.
}

\author{
Ankit Chakraborty ${ }^{1}$, Ajay Rangarajan ${ }^{1}$ and Georg May ${ }^{2}$ \\ ${ }^{1}$ RWTH Aachen University \\ Schinkelstrasse 2, Aachen, Germany \\ chakraborty,rangarajan@aices.rwth-aachen.de \\ ${ }^{2}$ Von Karman Institute for Fluid Dynamics \\ Waterloosesteenweg 72, Sint-Genesius-Rode 1640,Belgium \\ georg.may@vki.ac.be
}

Key words: Petrov-Galerkin Schemes, Optimal Test Functions, Anisotropic Mesh Adaptations

\begin{abstract}
In recent times, Petrov-Galerkin schemes with optimal test function framework have presented themselves as a stable and robust technique for solving partial differential equations. These schemes are also accompanied by an inbuilt error estimator, which makes them an ideal candidate for mesh adaptation. In this paper, we present a metric-based mesh adaptation strategy utilizing this inbuilt error estimator to generate optimal $h p$ meshes.
\end{abstract}

\section{Introduction}

Automatic mesh adaptation is a potent tool which aides the computation of efficient and accurate solution of partial differential equations. In this regard, metric-based anisotropic meshes and adaptive techniques have been very expedient for many applications, such as computational fluid dynamics $[1,2,3]$. When these adaptive techniques are combined with higher order approximation methods, they present themselves as a very powerful tool in terms of higher accuracy with reduced degrees of freedom. This has also been pointed out in the Workshop on Higher-Order CFD Methods [4]. In terms of robust and stable methods for solving partial differential equation, Petrov-Galerkin (PG) Schemes with optimal test functions have been a critical development in last decade $[5,6,7,8,9,10,11]$. Given the weak formulation of the underlying partial differential equation with a priori chosen approximation space, this framework aims to compute a space of test functions such that the scheme is stable. Another significant advantage of this framework is the presence of an inbuilt error estimator. With the inbuilt error estimators, these schemes are tailor-made for supporting mesh adaptation techniques.

In this paper, we propose a metric-based anisotropic adaptation strategy which uses this inbuilt error estimator to generate meshes with prescribed size and shape distribution along with a variable polynomial distribution for approximating the solution. The article is structured as follows. In section 2, we will briefly address PG schemes with optimal test functions. In section 3, we will describe the relationship between a metric field and a mesh. 
This is followed by anisotropy computations in section 4 and a continuous mesh model in section 5. Finally, in section 6 , we provide numerical results and concluding remarks in section 7 .

\section{Discretization}

In this section, we provide a brief description of PG schemes with optimal test functions and the associated inbuilt error estimator. We consider any inf-sup stable variational problem :

$$
u \in\left(U,\|\cdot\|_{U}\right): \quad\langle\mathcal{L} u, v\rangle=\langle f, v\rangle, \quad \forall v \in\left(\mathbb{V},\|\cdot\|_{\mathbb{V}}\right)
$$

where $\mathcal{L}: U \rightarrow \mathbb{V}^{\prime}, f \in \mathbb{V}^{\prime}, U$ and $\mathbb{V}$ are Hilbert spaces with $U^{\prime}$ and $\mathbb{V}^{\prime}$ being the respective dual spaces. One way to characterize PG Schemes with optimal test functions [6] is the following: Given $\|\cdot\|_{\mathbb{V}}$ and $\|\cdot\|_{U}$, they satisfy

$$
\|\mathcal{L} u\|_{\mathbb{V}^{\prime}}=\|u\|_{E}=\sup _{v \in \mathbb{V}} \frac{|\langle\mathcal{L} u, v\rangle|}{\|v\|_{\mathbb{V}}} \Rightarrow|\langle\mathcal{L} u, v\rangle| \leq\|u\|_{E}\|v\|_{\mathbb{V}}
$$

In a finite dimensional setting, given $U_{h} \subset U$, optimal PG schemes construct $\mathbb{V}_{h}^{\text {opt }}=$
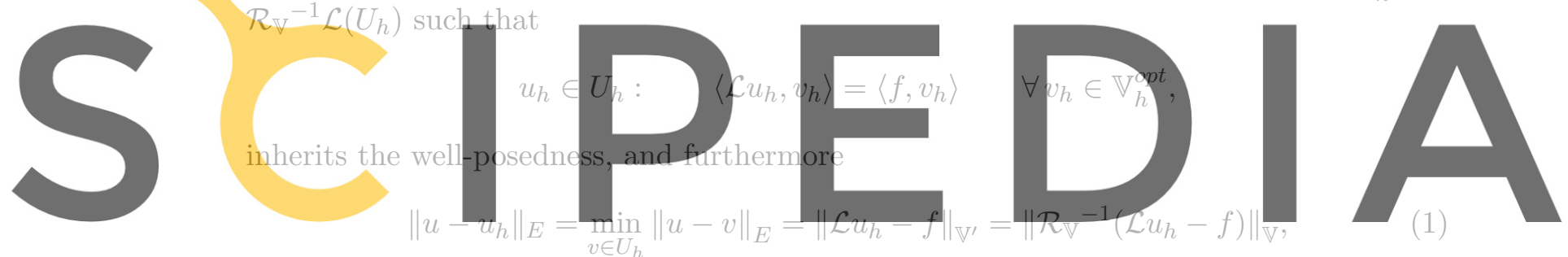

Register for free at https//wwws sfipedia. fom to download, the veksion, without the watermark

Since $\mathbb{V}$ is infinite-dimensional, it is not possible to explicitly compute $\mathcal{R}_{\mathbb{V}}^{-1}$. Instead, a choice of enriched space $\mathbb{V}_{r} \subset \mathbb{V}\left(M=\operatorname{aim}\left(\mathbb{V}_{r}\right) \geq \operatorname{aim}\left(U_{h}\right)=N\right)$ is employed over which the Riesz map is discretized and inverted. Since we are approximating the inverse of $\mathcal{R}_{\mathbb{V}}$, we have $\mathbb{V}_{h}$ which approximates $\mathbb{V}_{h}^{\text {opt }}$. It also implies $\mathbb{V}_{h} \subset \mathbb{V}_{r} \subset \mathbb{V}$. The discretization of the Riesz map is represented by a Gram matrix $\mathbb{G}_{i, j}=\left(\psi_{i}, \psi_{j}\right)_{\mathbb{V}}$ which is induced by the inner product on $\mathbb{V}$. Here $\psi_{i}$ and $\psi_{j}$ represent the basis of enriched space $\mathbb{V}_{r}$. The resulting discrete system can be shown to be equivalent to the following linear system [11]

$$
B^{T} \mathbb{G}^{-1} B \hat{x}_{h}=B^{T} G^{-1} l,
$$

where $u_{h}=\sum_{i=1}^{N}\left(\hat{x}_{h}\right)_{i} \phi_{i}$ is the discrete solution with $\phi_{i}$ being the basis of the finite dimensional trial space $U_{h} . B$ is the enriched stiffness matrix where $B_{i j}=\left\langle\mathcal{L} \phi_{j}, \psi_{i}\right\rangle$ and $l$ is the enriched load vector with $l_{i}=\left\langle f, \psi_{i}\right\rangle$. The residual based error estimator in eq. (1) can be approximated due to discretization of $\mathcal{R}_{\mathbb{V}}$ and is given by:

$$
\hat{y}=\mathbb{G}^{-1}\left(B \hat{x}_{h}-l\right),
$$

where the dual of residual is approximated as $\varphi=\sum_{i=1}^{M} \hat{y}_{i} \psi_{i}$, and hence the error in energy norm is approximated as $\left\|u-u_{h}\right\|_{E} \approx\|\varphi\|_{\mathbb{V}}$. The function $\varphi$ is also known as error representation function. In this article, we present results for scalar convection-diffusion problems using ultraweak formulation and discontinuous Galerkin discretization (for approximation spaces) with optimal test functions (DPG schemes) [6]. $U_{h}$ will represent 
the solution of the scalar convection-diffusion problem i.e. $U_{h}=\left(u_{h}, \sigma_{h}, \hat{u}_{h}, \hat{\sigma}_{h}\right)$. The numerical solution $u_{h}$ represents the approximation of primary variable $u, \sigma_{h}$ represents the approximation of gradient of $u, \hat{u}_{h}$ approximates the trace of $u$ and $\hat{\sigma}_{h}$ approximates the normal flux $\sigma \cdot \mathbf{n}$. The variables $\hat{u}_{h}$ and $\hat{\sigma}_{h}$ exists on mesh skeleton. Hence, for convectiondiffusion problems, the global energy error will be represented by $\left\|U-U_{h}\right\|_{E(\Omega)}$ and local energy error will be represented by $\left\|U-U_{h}\right\|_{E, k}$. Here, $\Omega$ represents the computational domain and $T_{h}$ represents its triangulation with $k$ being an element in $T_{h}$.

\section{Mesh-Metric Duality}

In this section, we briefly present the concept of metric-based mesh representation. For this, we will be aided by a tensor valued field $\mathcal{M}(\mathbf{x})$. Let $T_{h}$ be a given triangulation of $\Omega \in \mathbb{R}^{2}$. Each element in this $T_{h}$ can be characterized by a symmetric positive definite matrix in such a way that we can extract information about the shape and the size of the element by analysing this matrix. To be precise, let $e_{k}$ for $k=1,2,3$ represent the edges of a non-degenerate triangle $k \in T_{h}$. Then, there exists a symmetric positive definite Matrix $\mathcal{M} \in \mathbb{R}^{2 \times 2}$

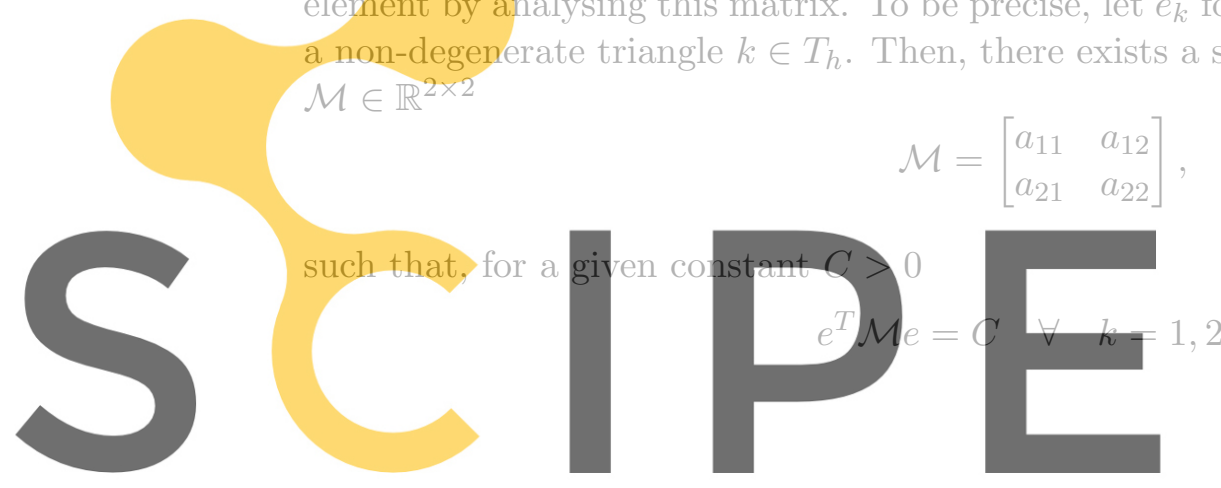

$$
\mathcal{M}=\left[\begin{array}{ll}
a_{11} & a_{12} \\
a_{21} & a_{22}
\end{array}\right]
$$

Register for free at https//www.scipedia.com to download the version without the watermark

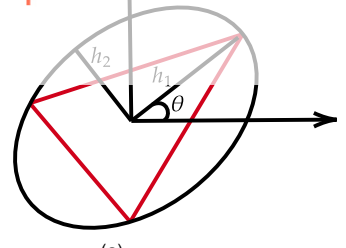

(a)
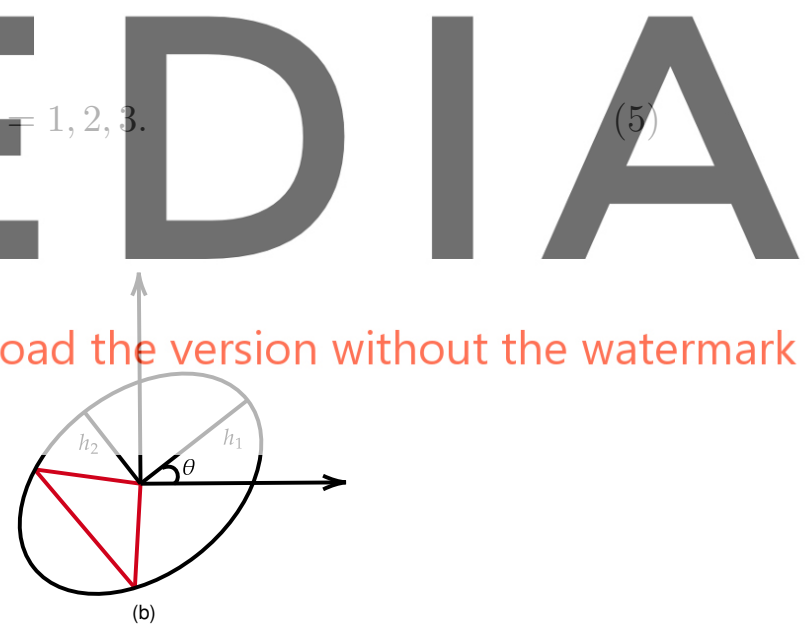

Figure 1: Ellipse circumscribing a triangle with (a) $\mathrm{C}=3$ and (b) $\mathrm{C}=1$

If $\mathcal{M}$ is defined at every $\mathbf{x} \in \Omega$, we call it as a metric field. From eq. (5), one can infer that the triangle is equilateral under the norm induced by $\mathcal{M}$ i.e. $\|\mathbf{x}\|_{\mathcal{M}}=\sqrt{\mathrm{x}^{T} \mathcal{M} \mathbf{x}}$. A mesh is called a unit mesh with respect to a metric field $\mathcal{M}(\mathbf{x})$ if every element in the mesh is equilateral with respect to $\mathcal{M}$.

As illustrated in fig. 1, a metric implies an ellipse, defined by three parameters, into which a unit element is inscribed. These parameters are the length of the major axis denoted by $h_{1}$, the minor axis denoted by $h_{2}$ and the orientation of the major axis denoted by $\theta$. On performing eigenvalue decomposition of $\mathcal{M}[12,13]$, one can observe how these quantities are encoded in the metric field. Indeed one can write

$$
\mathcal{M}=\left[\begin{array}{cc}
\cos (\theta) & -\sin (\theta) \\
\sin (\theta) & \cos (\theta)
\end{array}\right]^{T}\left[\begin{array}{cc}
\frac{1}{h_{1}^{2}} & 0 \\
0 & \frac{1}{h_{2}^{2}}
\end{array}\right]\left[\begin{array}{cc}
\cos (\theta) & -\sin (\theta) \\
\sin (\theta) & \cos (\theta)
\end{array}\right],
$$


The eigenvectors represent the principal axes of the ellipse whereas eigenvalues are inverse of the square of principle lengths $h_{1}$ and $h_{2}$ such that $h_{1}>h_{2}$. Next, we define the aspect ratio of an element as $\beta=\frac{h_{1}}{h_{2}}$. From the relation between the area of an ellipse and its corresponding triangle $(\mathrm{C}=3)$, we have:

$$
|k|=\frac{3 \sqrt{3}}{4} h_{1} h_{2}=\frac{3 \sqrt{3}}{4 d},
$$

where we define $d=1 / h_{1} h_{2}$ as the mesh density. We have introduced $d, \beta$ and $\theta$ to neatly separate the size and the anisotropy of the triangle. The dependence of eigenvalues and eigenvectors on $d, \beta$ and $\theta$ lays down the motivation of encoding the information for a desirable mesh into a metric field. This will be our focus in the next two sections. We have so far considered a constant metric field but the concept can be extended to a continuous Riemannian metric field. In that case, the norm induced by $\mathcal{M}(\mathbf{x})$ is given by $\|\mathrm{x}\|_{\mathcal{M}}=\int_{0}^{1} \sqrt{\mathrm{x}^{T} \mathcal{M}\left(\mathrm{x}_{1}+t \mathrm{x}_{2}\right) \mathrm{x}} d t$ where $\mathrm{x}:=\mathrm{x}_{2}-\mathrm{x}_{1}$. Such a Riemannian metric may be generated from a suitable interpolation resulting from the piecewise constant metric inferred from an existing mesh. There are already specific mesh generators which attempt to produce a metric-conforming from such a metric field. Examples of metric-based mesh generators are [14] and [15].

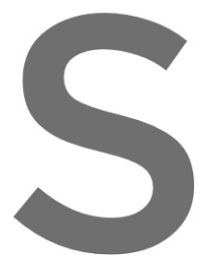

\section{Anisotropy

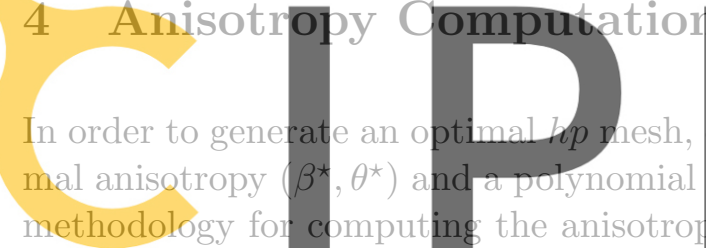

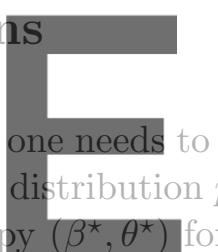
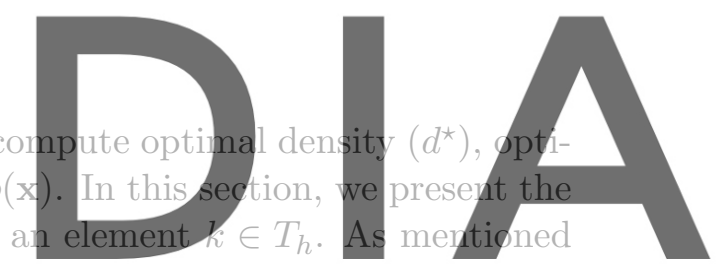

in section 2, DPG schemes with optimal test functions are accompanied with a residual

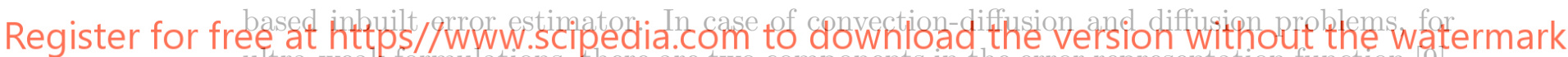
ultra-weak formulations, there are two components in the error representation function 9 .

We will denote them as $\left(\psi_{v}, \psi_{\tau}\right)$. In finite dimensional setting, we can approximate these error representation function using the same polynomial basis functions which are used to compute the near-optimal test space $\mathbb{V}_{h}$ (see section 2 ). This polynomial representation is very pivotal for anisotropy computation. Here, we demonstrate this with a scaled version of the norm induced by the inner product of $H^{1}\left(T_{h}\right) \times H\left(d i v ; T_{h}\right)$ (see [9] ):

$$
\begin{aligned}
\left\|\left(\psi_{v}, \boldsymbol{\psi}_{\tau}\right)\right\|_{V, k}^{2} & =\left\|\psi_{v}\right\|^{2}+\sqrt{|k|}\left\|\nabla \psi_{v}\right\|^{2}+\left\|\boldsymbol{\psi}_{\tau}\right\|^{2}+\sqrt{|k|}\left\|\nabla \cdot \boldsymbol{\psi}_{\tau}\right\|^{2} \\
& =\int_{k} \underbrace{\left.\left(\psi_{v}(\mathbf{x})\right)^{2}+\boldsymbol{\psi}_{\boldsymbol{\tau}}(\mathbf{x}) \cdot \boldsymbol{\psi}_{\tau}(\mathbf{x})+\sqrt{|k|}\left(\nabla \psi_{v}(\mathbf{x}) \cdot \nabla \psi_{v}(\mathbf{x})+\left(\nabla \cdot \boldsymbol{\psi}_{\tau}(\mathbf{x})\right)^{2}\right)\right)}_{e_{k}(\mathbf{x})} d \mathbf{x} .
\end{aligned}
$$

In eq. (8), $e_{k}(\mathbf{x})$ represents the polynomial which is being integrated to compute $\left\|\left(\psi_{v}, \boldsymbol{\psi}_{\tau}\right)\right\|_{V, k}^{2}$ and $|k|$ is the area of the element $k$. In [[16], Lemma 3.12 ], a very important result on an anisotropic bound is presented. We will just reiterate it next as it plays a fundamental role for the proposed anisotropy computation.

Lemma 1. Let $P_{i, \overline{\mathbf{x}}}(\mathbf{x}): \Omega \rightarrow \mathbb{R}$ be a homogeneous polynomial of order $i$ located at $\overline{\mathbf{x}}$, $i \geq 2$. Then there exist values $A_{i}, \rho_{i}$ and $\phi_{i} \in[0,2 \pi)$ such that

$$
\left|P_{i, \overline{\mathbf{x}}}(\mathbf{x})\right| \lesssim A_{i}\left((\mathbf{x}-\overline{\mathbf{x}})^{T} Q_{\phi_{i}} D_{\rho_{i}}{Q_{\phi_{i}}}^{T}(\mathbf{x}-\overline{\mathbf{x}})\right)^{\frac{i}{2}} .
$$


In lemma $1, D_{\rho_{i}}=\operatorname{diag}\left(1, \rho_{i}^{-2 / i}\right), Q_{\phi_{i}}$ is a $2 \times 2$ rotation matrix through an angle $\phi_{i}$ counter-clockwise and $A_{i}>0$. For a given polynomial $P_{i, \overline{\mathbf{x}}}$, the values of $A_{i}, D_{\rho_{i}}$ and $Q_{\phi_{i}}$ can be computed numerically in such a way that Lemma 1 is sharp. Details about this can be found in [16]. In case of $e_{k}(\mathbf{x})$, it is a combination of multiple homogeneous polynomials. For an order $p$ approximation for the solution variables with an enrichment of $\delta p$ for $\mathbb{V}_{r}$, there will be a total of $2(p+\delta p)$ sets of homogeneous polynomials. Since, the element $k \in T_{h}$ is inscribed in an ellipse which we will represent by $E_{k}$ and using Lemma 1, we have

$$
\int_{k} e_{k}(\mathbf{x}) d \mathbf{x} \leq \int_{E_{k}} e_{k}(\mathbf{x}) d \mathbf{x} \lesssim \sum_{i=2, i \in Z_{e v}^{+}}^{2(P+\delta P)} \int_{E_{k}} A_{i}\left(\left(\mathbf{x}-\overline{\mathbf{x}}_{k}\right)^{T} Q_{\phi_{i}} D_{\rho_{i}}{Q_{\phi_{i}}}^{T}\left(\mathbf{x}-\overline{\mathbf{x}}_{k}\right)\right)^{\frac{i}{2}} d \mathbf{x},
$$

where $Z_{e v}^{+}$represents the set of positive even integers. The subscript $e v$ represents the even nature of the integers. Since, integrals of odd order homogeneous polynomial is zero over a symmetric interval about origin (here $\overline{\mathbf{x}}_{k}$ can be treated as the origin), we are left with only even ordered polynomials. A mapping $F: \hat{E} \rightarrow R^{2}$ can be defined as $F(\hat{\mathbf{x}})=Q_{e} S_{e} \hat{\mathbf{x}}+\overline{\mathbf{x}}_{k}$ where $\hat{E}$ represents the unit circle, $Q_{e}$ is a rotation matrix through an angle $\phi$ and $S_{e}=\operatorname{diag}\left(h_{1}, h_{2}\right)=h_{1} \operatorname{diag}(1,1 / \beta)$ where $\beta$ is the aspect ratio. On introducing this transformation $(\mathbf{x}=F(\hat{\mathbf{x}}))$ into eq. $(10)$ and polar co-ordinates $\hat{\mathbf{x}}=\left[\begin{array}{ll}\cos (\theta) & \sin (\theta)\end{array}\right]^{T}$, we have
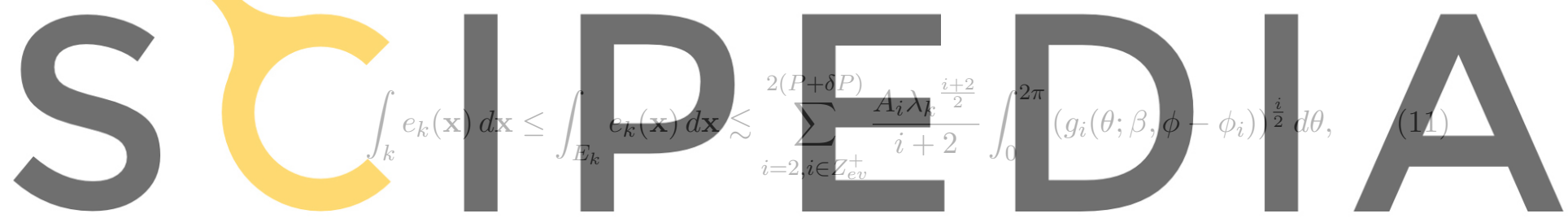

where $\lambda_{k}=\frac{1}{d_{k}}$ and $d_{k}$ is computed using the implied metric (computed using the vertices

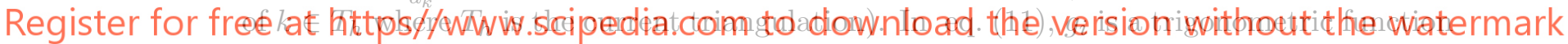
which is obtained after performing some arithmetic following the substitution mentioned earlier. The expression for $g_{i}$ can be be found in [17] and [18]. Hence, the computation of the anisotropy becomes a minimization problem.

$$
\beta^{\star}, \phi^{\star}=\underset{\beta, \phi}{\operatorname{argmin}} \sum_{i=2, i \in Z_{\text {ev }}^{+}}^{2(P+\delta P)} \frac{A_{i} \lambda_{k}{ }^{\frac{i+2}{2}}}{i+2} \int_{0}^{2 \pi}\left(g_{i}\left(\theta ; \beta, \phi-\phi_{i}\right)\right)^{\frac{i}{2}} d \theta .
$$

The above minimization problem has been solved using an iterative process mentioned in [18] and [17].

\section{Continuous model of energy error estimate for $h p$ adap- tation}

In this section, we present the procedure for $h p$ adaptations using the inbuilt energy error estimator that accompanies DPG schemes with optimal test functions. The procedure is a two-step process that comprises of selecting a polynomial order for approximation followed by a mesh density computation $(d(\mathbf{x}))$ based on the concept of continuous mesh. The following assumption is fundamental for mesh density computation. 
Assumption 5.1: Let $T_{h}$ be a triangulation and $\eta$ be a local error estimate such that $\eta^{2}=\sum_{k \in T_{h}} \eta_{k}^{2}$ and $\eta=O\left(h^{s}\right)$ i.e error estimate converges at $s^{t h}$ order [19]. We assume that local error estimate $\eta_{k}$ scales ${ }^{1}$ as $\eta_{k}^{2}=\bar{A}|k|^{(s+1)}$ where $\bar{A}$ depends upon the anisotropy of the element. Furthermore, the order of the method $s$ directly depends upon the polynomial degree of approximation $p$.

Verification of assumption 5.1 can be found in [section 8.1, [19]] and [17] where the authors have demonstrated this with a Laplace problem having an exact solution $u$ of the form $u=\sin (2 \pi x) \sin (2 \pi y)$ and in [section 3.2, [20]] where similar properties are studied for compressible flow problems. Let $\left\{T_{h}\right\}_{n}$ be the sequence of triangulation employed, we would like to construct an error estimate which achieves global equality with the inbuilt energy error estimate asymptotically.

$$
\left\|U-U_{h}\right\|_{E, k}^{2} \approx e_{d}\left(\mathbf{x}_{k}\right)|k| \quad \text { for } \mathbf{x}_{k} \in k, k \in T_{h},
$$

\section{where we call $e_{d}\left(\mathbf{x}_{k}\right)$ as the error density function. Thus, asymptotically $(h \rightarrow 0)$, we have}

$$
\left\|U-U_{h}\right\|_{E(\Omega)}^{2}=\sum_{k \in T_{h}} e_{d}\left(\mathbf{x}_{k}\right)|k| \rightarrow \int_{\Omega} e_{d}(\mathbf{x}) d \mathbf{x} .
$$

\section{Next, we define the error density function as following}
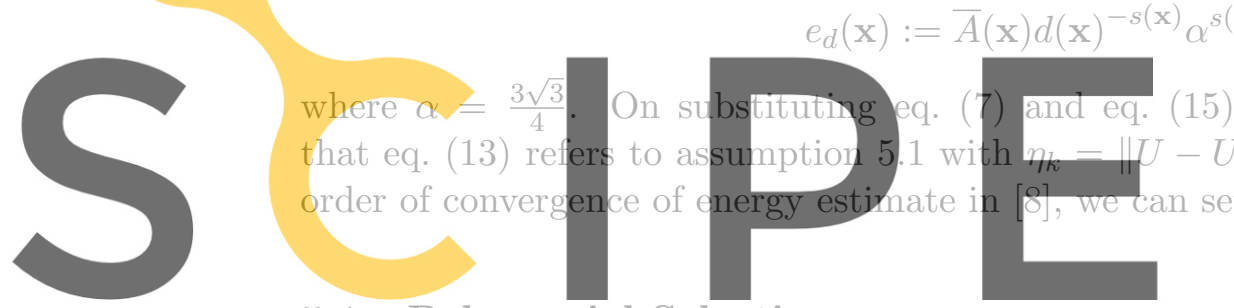

\subsection{Polynomial Selection}

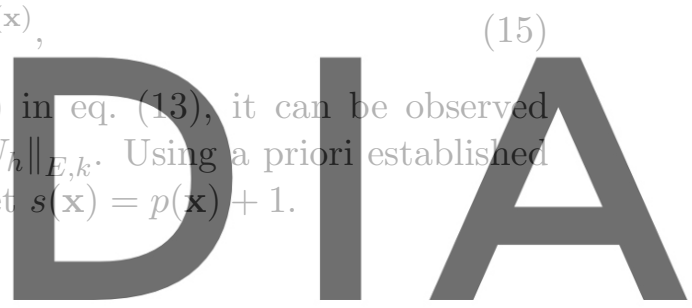

Register for free at https//www.scipedia.com to download the version without the watermark In order to choose the proper polynomial order for approximation, we solve the governing partial differential equation locally over a patch surrounding an element $k \in T_{h}$. The boundary conditions for these local problems are obtained using trace $\left(\hat{u}_{h}\right)$ in case of Dirichlet boundary conditions or normal flux $\left(\hat{\sigma}_{h}\right)$ for Nuemann boundary condition computed at the current polynomial order. In fig. 2, we have shown an internal patch where we have used Dirichlet boundary condition. We solve these local problems at three different polynomial orders: $p_{k}$ and $p_{k} \pm 1$ where $p_{k}$ represents the current polynomial order of the element $k$. Once the solution is computed, it is followed by computing the local energy error estimate $\left\|U-U_{h}\right\|_{E, k}$ for each polynomial order (the local problem is also solved at $p_{k}$ so that the error at $p_{k}$ has similar fidelity as the error computed for $\left.p_{k} \pm 1\right)$. We denote the energy error computed at the polynomial order $p_{k}+i$ by $E_{p_{k}+i}$ and the number of degrees of freedom for each polynomial order by $N_{p_{k}+i}$ where $i=-1,0,1$. Also, we introduce a new parameter $m_{p_{k}+i}$ which corresponds to the amount of uniform refinement or coarsening required to achieve a level of error. The motivation behind this is to use the a priori rate of convergence to predict the required uniform coarsening or refinement at a local level. It is computed as follows:

$$
m_{p_{k}+i}=\left(\frac{E_{p_{k}+i}}{E_{p_{k}}}\right)^{\frac{2}{p_{k}+i+1}} N_{p_{k}+i}
$$

\footnotetext{
${ }^{1}$ To motivate the scaling $\eta_{k}^{2} \propto|k|^{(s+1)}$, recall that the global error estimate $\eta^{2}$ scales as $h^{2 s} \propto|k|^{s}$. (we have a $s^{t h}$ order method.) At the same time, the contribution from each individual sub-element scales with an additional factor of $k$, because the local domain of integration becomes smaller.
} 


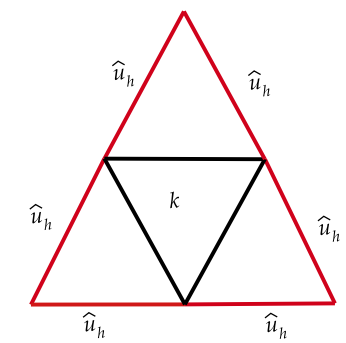

Figure 2: Patch around an element $k$ over which local problems are solved. Edges marked by red represents the boundaries of patch with Dirichlet boundary conditions

We have taken the a priori rate of convergence to be $\left(p_{k}+i\right)+1$ where $i=-1,0,1$. In order to choose a better suited polynomial order, we computed $m_{p_{k}+i}$ for $p_{k}-1$ and $p_{k}+1$ to achieve the same error as $p_{k}$ and optimal order is the one which achieves $E_{p_{k}}$ with least degrees of freedom.

$$
p_{k, o p t}=\underset{i=-1,0,1}{\operatorname{argmin}} m_{p_{k}+i}
$$

The idea behind this is to find out the polynomial order which is more efficient in terms of number of degrees of freedom to the achieve the same level of error as $p_{k}$. For $i=0$, above computation is trivial. Once the polynomial order is selected, we still need to compute
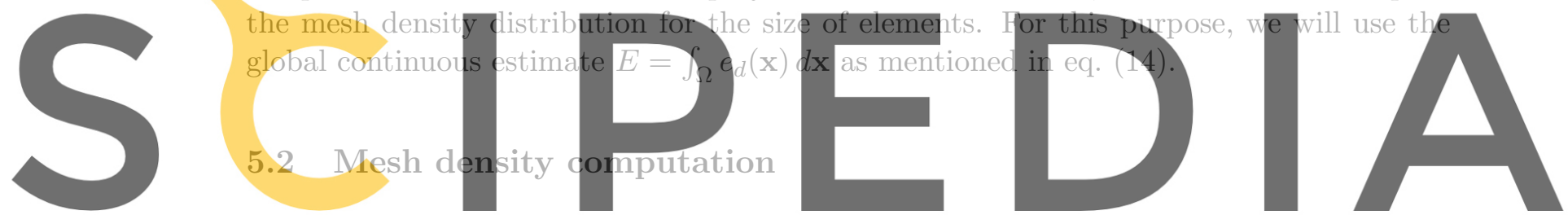

In order to generate the optimal density distribution at fixed cost, we need to have a notion

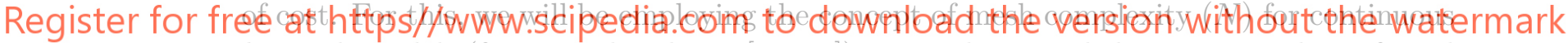

$h p$ mesh models (for more details see [21, 22]). It can be regarded as an equivalent of total degrees of freedom desired in a mesh.

Problem 5.1: Let $N$ be the desired complexity in terms of degrees of freedom and $e_{d}(\mathbf{x})$ be the error density function. We seek a mesh density distribution $d(\mathbf{x}): \Omega \rightarrow \mathbb{R}^{+}$for a given polynomial distribution $p(\mathbf{x}): \Omega \rightarrow Z^{+}$on the next triangulation such that:

(a) $N=\int_{\Omega} w(\mathbf{x}) d(\mathbf{x}) d \mathbf{x} \quad$ with $\quad w(\mathbf{x})=\frac{2(p(\mathbf{x})+1)(p(\mathbf{x})+2)}{3 \sqrt{3}}$.

(b) $E=\int_{\Omega} e_{d}(\mathbf{x}) d \mathbf{x}$ is minimized.

For density computation, we have implemented the analytic optimization for $h p$ continuous mesh model mentioned in [22]. Using eq. (15), we state the global continuous error as follows:

$$
E=\int_{\Omega} \alpha^{(p(\mathbf{x})+1)} \bar{A}(\mathbf{x}) d(\mathbf{x})^{-(p(\mathbf{x})+1)} d \mathbf{x} .
$$

In case of $h p$ adaptivity, the constraint is the total number of degrees of freedom desired in the next mesh which is obtained by multiplying a weight to mesh density distribution $[21,22]$.

$$
N=\int_{\Omega} w(\mathbf{x}) d(\mathbf{x}) d \mathbf{x} \quad \text { with } \quad w(\mathbf{x})=\frac{2(p(\mathbf{x})+1)(p(\mathbf{x})+2)}{3 \sqrt{3}} .
$$


Taking variation of global continuous error and complexity constraint with respect to density, we get

$$
\begin{gathered}
\delta E=\int_{\Omega}-(p(\mathbf{x})+1) \alpha^{(p(\mathbf{x})+1)} \bar{A}(\mathbf{x}) d^{-(p(\mathbf{x})+2)} \delta d d \mathbf{x} \\
\delta N=\int_{\Omega} w(\mathbf{x}) \delta d(\mathbf{x}) d \mathbf{x}=0 .
\end{gathered}
$$

Using the eq. (19), it can be observed that we can obtain an admissible solution if we set

$$
\frac{(p(\mathbf{x})+1) \bar{A}(\mathbf{x})}{w(\mathbf{x})} \alpha^{(p(\mathbf{x})+1)} d(\mathbf{x})^{-(p(\mathbf{x})+2)}=K=\text { const } .
$$

Solving this for $d(\mathbf{x})$ vields

$$
d^{\star}(\mathbf{x})=\left(\frac{(p(\mathbf{x})+1) \bar{A}(\mathbf{x}) \alpha^{(p(\mathbf{x})+1)}}{w(\mathbf{x})}\right)^{\frac{1}{(p(\mathbf{x})+2)}} K^{-\frac{1}{(p(\mathbf{x})+2)}} .
$$

The constant $K$ can be computed from eq. (19) numerically. In our current implementation, we employ bisection method to compute $K$. Once $K$ is computed, we can substitute
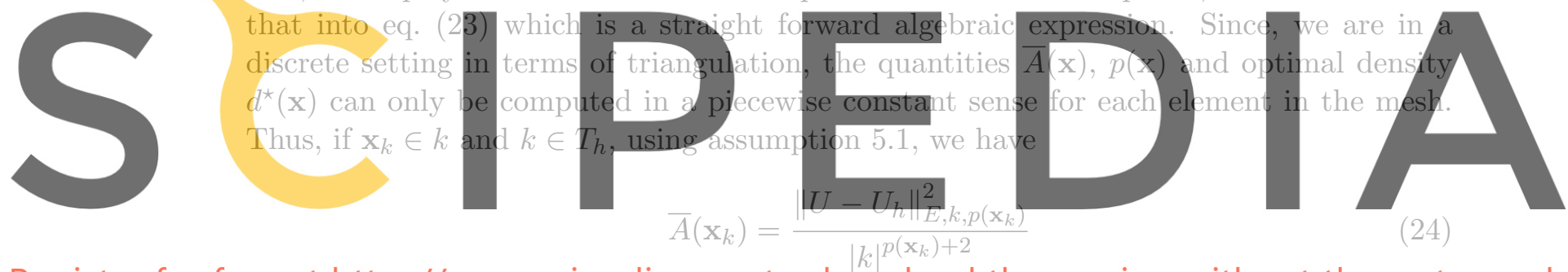

(24)

Register for free at https//www.scipedia.com to download the version without the watermark

$$
d^{\star}\left(\mathbf{x}_{k}\right)=\left(\frac{\left(p\left(\mathrm{x}_{k}\right)+1\right) \bar{A}\left(\mathrm{x}_{k}\right) \alpha^{\left(p\left(\mathrm{x}_{k}\right)+1\right)}}{w\left(\mathbf{x}_{k}\right)}\right)^{\frac{1}{\left(p\left(x_{k}\right)+2\right)}} K^{-\frac{1}{\left(p\left(\mathbf{x}_{k}\right)+2\right)}}
$$

where $\left\|U-U_{h}\right\|_{E, k, p\left(\mathbf{x}_{k}\right)}$ represents the energy error in element $k$ for the optimal polynomial order chosen via the process mentioned in section 5.1.

\section{$6 \quad$ Results}

Sharp boundary layers are one of the most encountered features in flow fields. This test problem is selected in order to validate the fidelity of the proposed algorithm in presence of boundary layers. In particular, we solve,

$$
\begin{aligned}
\beta \cdot \nabla u-\epsilon \nabla^{2} u & =s(x, y) & & (x, y) \in \Omega=(0,1)^{2} \\
u & =0 & & (x, y) \in \partial \Omega
\end{aligned}
$$

where $\beta=[1,1]^{T}$. The source term $s(x, y)$ is selected in such a way that the exact solution is given by

$$
u(x, y)=\left(x+\frac{e^{\frac{x}{\epsilon}}-1}{1-e^{\frac{1}{\epsilon}}}\right)\left(y+\frac{e^{\frac{y}{\epsilon}}-1}{1-e^{\frac{1}{\epsilon}}}\right) .
$$


The solution exhibits a sharp boundary layer near $x \approx 1, y \approx 1$, increasingly so for smaller values of $\epsilon$. In fig. 4, we have presented the convergence results comparing the $h$ adaptation algorithm [17] and proposed $h p$ adaptation algorithm. In $h$ adaptations, we start with an initial mesh of 32 elements and in case of $h p$ adaptations, we begin with the same mesh but with constant initial polynomial order of $p_{\text {initial }}=2$. In $h p$ adaptation, $N$ for the first adaptation cycle is computed as

$$
N=N e \times \frac{\left(p_{\text {initial }}+1\right)\left(p_{\text {initial }}+2\right)}{2} \times \frac{3 \sqrt{3}}{4}
$$

where $N e$ represents the number of elements in the mesh. Between each adaptation cycle, $N$ is increased by $30 \%$. This choice of growth in $N$ is arbitrary and different choice in growth may result in different pre-asymptotic behaviour but should produce similar asymptotic result.
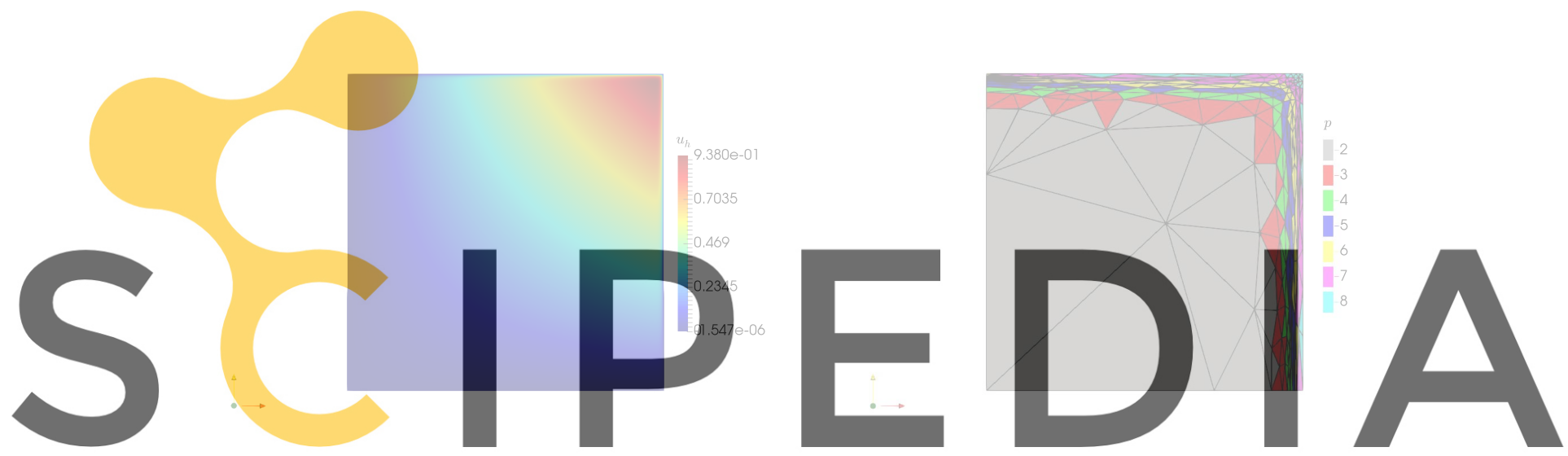

Register for free at https//www.scipedia.com to download the version without the watermark

Figure 3: Boundary Layer: (a) Solution contour on an adapted mesh and (b) polynomial distribution on the same adapted mesh with $\epsilon=0.005$ with 6980 degrees of freedom.

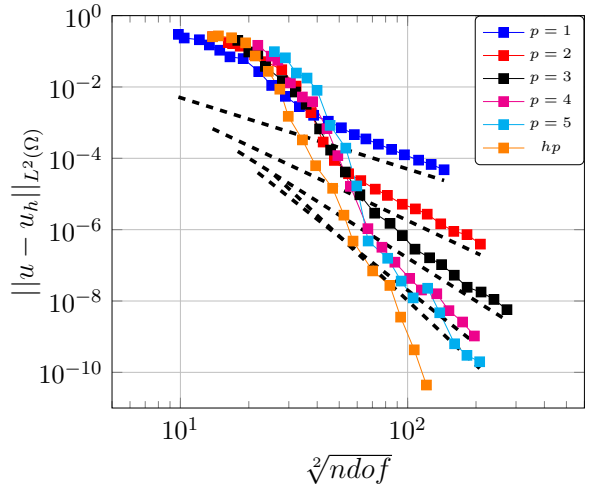

(a)

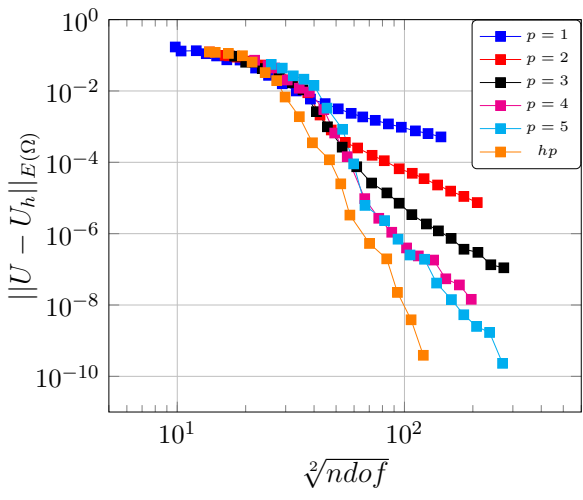

(b)

Figure 4: Convergence plots of (a) $L^{2}$ error in $u_{h}$ and (b) Energy norm using scaled mathematician's norm (ndof represents the total number of degrees of freedom). 


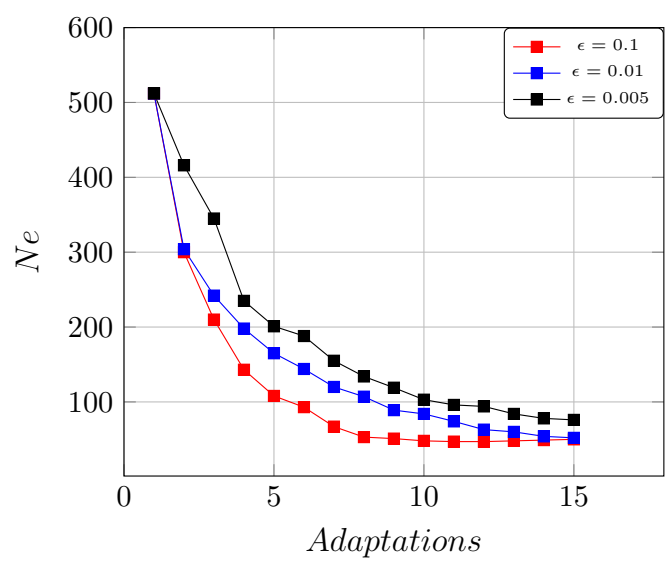

(a)

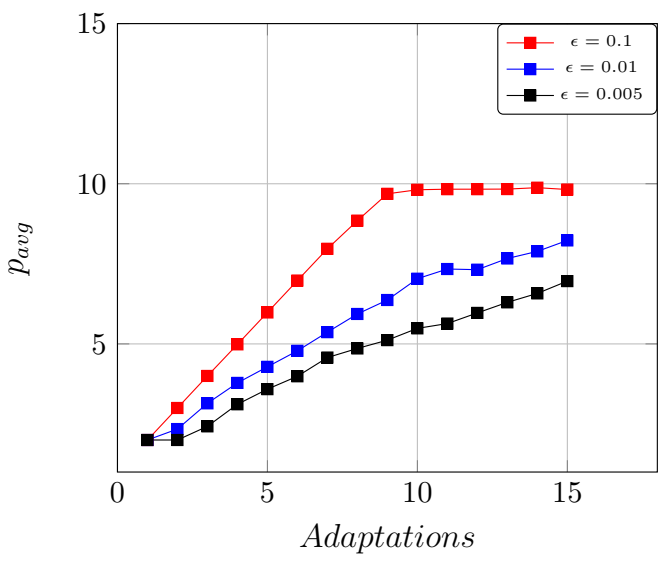

(b)

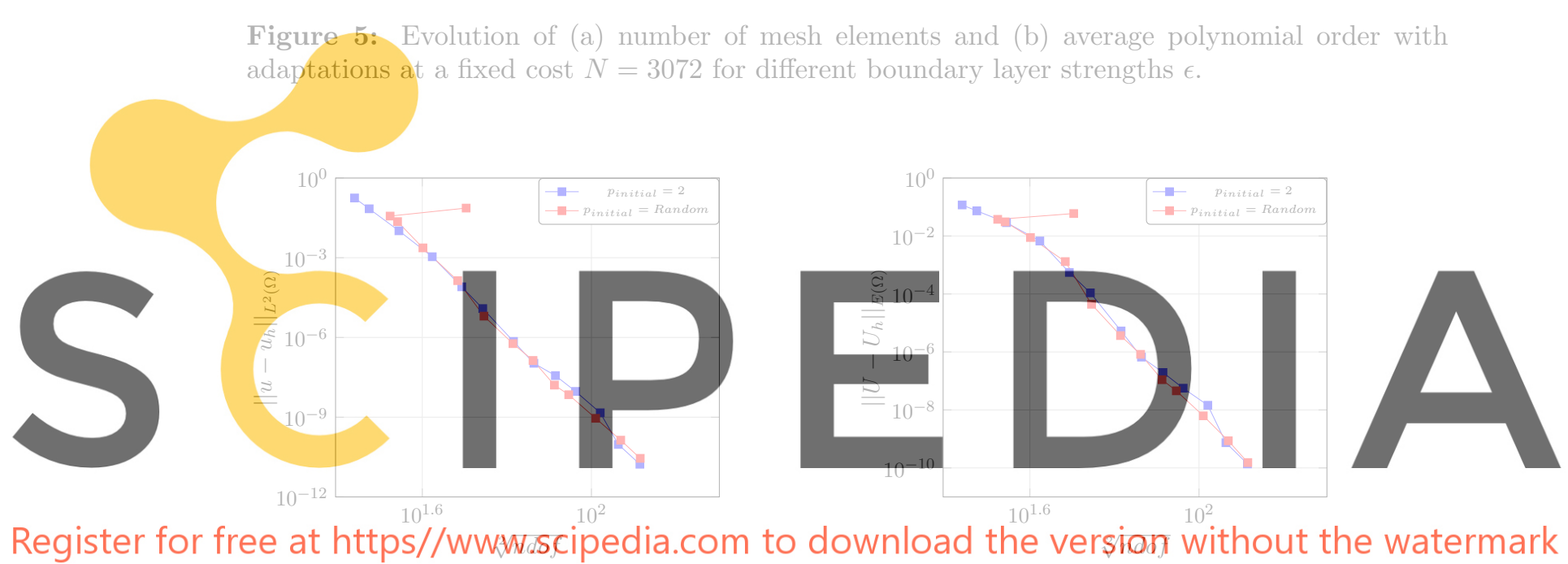

(a)

(b)

Figure 6: Convergence plots of (a) $L^{2}$ error in $u_{h}$ and (b) Energy norm using scaled mathematician's norm with different initial polynomial distributions (ndof represents the total number of degrees of freedom).

In $h p$ adaptations, we have kept an upper limit on the highest possible polynomial order as $p_{\max }=10$. Since, the analytical solution is in $C^{\infty}$, there is no theoretical limit on the highest allowable polynomial order. This limit is prescribed for the practical computations [23]. On continuously increasing $N$, the $h p$ algorithm produces a better convergence plot compared to the $h$ adaptation at constant polynomial order $p=5$ in terms of convergence rate as shown in fig. 4 . In $h p$ adaptation, initially on increasing $N$ the adaptation is dominated by $h$ refinement. Consequently once the boundary layer is resolved, $p$ adaptation takes over and $h p$ algorithm selects maximum polynomial order in almost every cell in the boundary layer whereas it prescribes $p=2$ away from the boundary layer. Away from the boundary layer the analytical solution is nearly quadratic, at least up to machine precision, i.e. $u(x) \approx x y$. Since with the assumption of quasi-regular elements, one can show the equivalence of error in energy norm (using the inner product on the test space induced by the norm given by eq. (8)) with the error in $L^{2}$ norm of the field variables, one can expect to obtain the polynomial distribution reflecting the local regularity of the solution. The evolution of average polynomial order with subsequent adaptations is shown 
in fig. 5. In fig. 6 , we show how two different initial polynomial distribution have similar asymptotic convergence behaviour. This reflects the robustness of the algorithm towards the user-controlled parameter of selecting the polynomial order on the initial mesh.

\section{Conclusions}

We have presented a continuous $h p$ mesh model for driving mesh adaptations which solely depends upon the inbuilt error estimator accompanying the numerical scheme rather than depending on an extrinsic error model. One major advantage of the proposed methodology is being parameter free. In the future, we would like to extend this $h p$ framework to system of equations and goal-oriented adaptations.

\section{Acknowledgement}

We would like to thank the Deutsche Forschungsgemeinschaft (DFG). This work was funded by the Deutsche Forschungsgemeinschaft (DFG, German Research Foundation) $33849990 /$ GRK2379 (IRTG Modern Inverse Problems).

\section{REFERENCES}

[1] Marco Ceze and Krzysztof J. Fidkowski. Anisotropic hp-adaptation framework for functional prediction. AIAA Journal, 51(2):492-509, 2013.

[2] Tobias Leicht and Ralf Hartmann. Anisotropic mesh refinement for discontinuous galerkin methods in two-dimensional aerodynamic flow simulations. International Journal for Numerical Methods in Fluids, 56(11):2111-2138, 2008.

[3] Tobias Leicht and Ralf Hartmann. Error estimation and anisotropic mesh refinement for 3d laminar aerodynamic flow simulations. Journal of Computational Physics, 229(19):7344 - 7360, 2010.

[4] Z. Wang, Krzysztof Fidkowski, Rémi Abgrall, Francesco Bassi, Doru Caraeni, Andrew Cary, H. Deconinck, Ralf Hartmann, Koen Hillewaert, H.T. Huynh, Norbert Kroll, Georg May, Per-Olof Persson, Bram van Leer, and Miguel Visbal. High-order cfd methods: Current status and perspective. International Journal for Numerical Methods in Fluids, 72:811-845, 012013.

[5] L. Demkowicz and J. Gopalakrishnan. A class of discontinuous Petrov-Galerkin methods. Part I: The transport equation. Computer Methods in Applied Mechanics and Engineering, 199(23-24):1558-1572, 2010.

[6] L. Demkowicz and J. Gopalakrishnan. A class of discontinuous petrov-galerkin methods. ii. optimal test functions. Numerical Methods for Partial Differential Equations, 27(1):70-105, 2011.

[7] W. Dahmen, C. Huang, C. Schwab, and G. Welper. Adaptive Petrov-Galerkin methods for first order transport equations. SIAM J. Num. Anal., 50(5):2420-2445, 2012. 
[8] L Demkowicz and J Gopalakrishnan. Analysis of the dpg method for the poisson equation. SIAM Journal on Numerical Analysis, 49:1788-1809, 2011.

[9] L Demkowicz, J Gopalakrishnan, and A Niemi. A class of discontinuous PetrovGalerkin methods. Part III: Adaptivity. Applied Numerical Mathematics, 62:396-427, 2013.

[10] Brendan Keith, Federico Fuentes, and Leszek Demkowicz. The DPG methodology applied to different variational formulations of linear elasticity. Computer Methods in Applied Mechanics and Engineering, 309:579-609, 2016.

[11] Ali Vaziri Astaneh, Federico Fuentes, Jaime Mora, and Leszek Demkowicz. High-order polygonal discontinuous Petrov-Galerkin (PolyDPG) methods using ultraweak formulations. Computer Methods in Applied Mechanics and Engineering, 332(July):686$711,2018$.

[12] A. Loseille and F. Alauzet. Continuous mesh framework part i: Well-posed continuous interpolation error. SIAM Journal on Numerical Analysis, 49(1):38-60, 2011.

[13] A. Loseille and F. Alauzet. Continuous mesh framework part ii: Validations and applications. SIAM Journal on Numerical Analysis, 49(1):61-86, 2011.

[14] F Hecht. BAMG: Bidimensional anisotropic mesh generator. 2006.

[15] Vit Dolejsi. ANGENER,version 3.1.

[16] Vít Dolej. Anisotropic hp adaptive method based on interpolation error estimates in the Lq norm. 82(13):80-114, 2014.

[17] Ankit Chakraborty, Ajay Rangarajan, and Georg May. Optimal approximation spaces for discontinuous petrov-galerkin finite element methods. 2020.

[18] Vít Dolejší, Georg May, Ajay Rangarajan, and Filip Roskovec. A Goal-Oriented HighOrder Anisotropic Mesh Adaptation Using Discontinuous Galerkin Method for Linear Convection-Diffusion-Reaction Problems. SIAM Journal on Scientific Computing, 41(3):A1899-A1922, 2019.

[19] Vit Dolejsi, Georg May, Filip Roskovec, and Pavel Solin. Anisotropic hp-mesh optimization technique based on the continuous mesh and error models. Computers \& 8 Mathematics with Applications, 74(1):45-63, 2017.

[20] David A. Venditti and David L. Darmofal. Grid adaptation for functional outputs: Application to two-dimensional inviscid flows. Journal of Computational Physics, 176(1):40 - 69, 2002.

[21] Ajay Rangarajan, Georg May, and Vit Dolejsi. Adjoint-based anisotropic hpadaptation for discontinuous galerkin methods using a continuous mesh model. Journal of Computational Physics, 409:109321, 2020.

[22] Vít Dolejší, Georg May, and Ajay Rangarajan. A continuous hp-mesh model for adaptive discontinuous Galerkin schemes. Applied Numerical Mathematics, 124(September):1-21, 2018.

[23] William F. Mitchell. How high a degree is high enough for high order finite elements? Procedia Computer Science, 51:246-255, 2015. International Conference On Computational Science, ICCS 2015. 